\title{
Atuação da enfermagem na prevenção da hipertensão arterial
}

\author{
Nursing performance in the prevention of arterial hypertension \\ Desempeño de enfermería en la prevención de la hipertensión arterial
}

Amanda Karem Lopes Lima ${ }^{1 *}$, Jackelliny Carvalho Neves ${ }^{1}$, Luciane Sousa Pessoa Cardoso ${ }^{1}$, Andressa Arraes Silva ${ }^{1}$, Larissa Silva Oliveira ${ }^{1}$, Railda Lima Rodrigues ${ }^{1}$, Joelmara Furtado dos Santos Pereira ${ }^{2}$, Rafaela Duailibe Soares ${ }^{2}$, Andréa Dutra Pereira², Lívia Alessandra Gomes Aroucha².

\section{RESUMO}

Objetivo: Identificar diante da atuação da enfermagem, os desafios na prevenção da Hipertensão Arterial na Atenção Primária de Saúde Métodos: Trata-se de um estudo transversal descritivo, com abordagem qualitativa realizado com os enfermeiros das unidades básicas de saúde selecionadas. Foi utilizado um questionário com perguntas referentes a temática. Resultados: Identificou-se os principais desafios da enfermagem diante da prevenção da hipertensão que são: conscientizar a população a manter hábitos de vida saudável; dificuldade em atingir toda a população com educação em saúde, assim como, dificuldade em trazer a população à UBS. Dentre os fatores que contribuem para a dificuldade de prevenir a hipertensão encontrou-se: obesidade, alimentação inadequada, sedentarismo, tabagismo, alcoolismo, fatores genéticos, fatores socioeconômicos. Conclusão: Nota-se que a enfermagem possui conhecimentos suficientes para orientar os pacientes a realizarem a prevenção da hipertensão. Porém, a população apresenta bastante resistência em seguir as orientações de enfermagem, pois mesmo possuindo consciência sobre os hábitos inadequados, sentem-se desmotivados às mudanças, em virtude da cronicidade da doença.

Palavras-chave: Hipertensão, Prevenção, Assistência de enfermagem.

\begin{abstract}
Objective: To identify the challenges in the prevention of hypertension in Primary Health Care in relation to nursing practice. Methods: This is a descriptive cross-sectional study with a qualitative approach, carried out with nurses from the selected basic health units. A questionnary with questions related to theme was used. Results: The main challenges faced by nurses in preventing hypertension were identified, which are: making the population aware of maintaining healthy living habits; difficulty in reaching the entire population with health education, as well as difficulty in bringing the population to the PHCU. Among the factors that contribute to the difficulty of preventing hypertension were found: obesity, inadequate diet, sedentary lifestyle, smoking, alcoholism, genetic factors, and socioeconomic factors. Conclusion: It can be seen that nursing has enough knowledge to guide patients to prevent hypertension. However, the population presents a lot of resistance in following the nursing orientations, because even though they are aware of the inadequate habits, they feel unmotivated to change, due to the chronicity of the disease.
\end{abstract}

Keywords: Hypertension, Prevention, Nursing care.

\section{RESUMEN}

Objetivo: Identificar los desafíos en la prevención de la hipertensión en la Atención Primaria de Salud ante el desempeño de la enfermería. Métodos: Se trata de un estudio descriptivo transversal con enfoque cualitativo realizado con enfermeras de las unidades básicas de salud seleccionadas. Se utilizó un cuestionario con preguntas relacionadas con el tema. Resultados: Se identificaron los principales retos de la enfermería en la prevención de la hipertensión, que son: la concienciación de la población para mantener hábitos de vida saludables; la dificultad para llegar a toda la población con la educación sanitaria, así como la dificultad para acercar a la población a la UBS. Entre los factores que contribuyen a la dificultad de prevenir la hipertensión se encontraron: la obesidad, la mala alimentación, el sedentarismo, el tabaquismo, el alcoholismo, los factores

1 Universidade Estadual do Maranhão (UEMA), Bacabal - MA. *E-mail: amandakll@outlook.com

2 Universidade Federal do Maranhão (UFMA), São Luis - MA. 
genéticos, los factores socioeconómicos. Conclusión: Por lo tanto, se observa que la enfermería posee conocimientos suficientes para orientar a los pacientes a realizar la prevención de la hipertensión. Por otra parte, la población se resiste bastante a seguir las orientaciones de la medicina, ya que, aun siendo conscientes de los hábitos inadecuados, se sienten desmotivados por los cambios, en virtud de la cronicidad de la enfermedad.

Palabras clave: Hipertensión, Prevención, Cuidados de enfermería.

\section{INTRODUÇÃO}

A Hipertensão Arterial Sistêmica (HAS), usualmente chamada de pressão alta é uma doença crônica multifatorial, resultante de complexas interações entre fatores genéticos e ambientais, atingindo milhares de pessoas no Brasil. Afetando em maior número os idosos, após os 55 anos, devido a perda da elasticidade das artérias e diminuição da complacência vascular em decorrência do processo de envelhecimento (REIS LLM, et al., 2018).

A estimativa de pessoas com hipertensão é de 600 milhões com crescimento global de $60 \%$ dos casos até 2025, estimando-se cerca de 7,1 milhões de mortes anuais, levando a um aumento dos custos ao Sistema de Saúde, com forte impacto socioeconômico (MALTA DC, et al., 2018).

Sendo caracterizada pela presença de níveis tensionais permanentemente elevados acima do limite da normalidade, descrita como uma situação clínica onde o indivíduo apresenta valores de pressão arterial, igual ou maior que $140 \times 90 \mathrm{~mm} \mathrm{Hg}$, resultando em uma sobrecarga ao coração que irá aumentar seu esforço para fazer a distribuição do sangue para todo o corpo, favorecendo o aparecimento de outras patologias devido a lesões causadas pela força exercidas nas paredes dos vasos (SOCIEDADE BRASILEIRA DE CARDIOLOGIA, 2016).

Segundo Dantas RCO e Roncalli AG (2019) na maioria dos casos a hipertensão não apresenta sintomatologia o que leva a uma diminuição nas taxas de prevenção e dificuldades no diagnóstico precoce. É uma condição hereditária em $90 \%$ dos casos, entretanto há vários fatores que influenciam nos níveis de pressão arterial, como os hábitos de vida do indivíduo, sendo eles o consumo de bebidas alcoólicas, obesidade, estresse, consumo demasiado de sal, níveis altos de colesterol, fatores socioeconômicos e sedentarismo. Além de fatores de risco não modificáveis como a genética, idade, sexo e etnia (PEREIRA DS, et al., 2017).

Atualmente encontra-se um número expressivo da incidência de hipertensão segundo dados do Sistema de Vigilância de Fatores de Risco e Proteção para Doenças Crônicas por Inquérito Telefônico (VIGITEL) $24,7 \%$ dos indivíduos habitantes nas capitais brasileiras apresentam diagnóstico de hipertensão, sendo a população idosa a mais afetada, $60,9 \%$ dos entrevistados com idade acima de 65 anos apresentam diagnostico de hipertensão, assim como 49,5\% na faixa etária de 55 a 64 anos (BRASIL, 2018). O aumento da perspectiva de vida da população, acarreta em um aumento da população idosa. $O$ processo de envelhecimento favorece o aparecimento de diversas alterações naturais que geram maior vulnerabilidade aos indivíduos, principalmente ao desenvolvimento de doenças crônicas não transmissíveis, entre elas a hipertensão arterial (MENEZES TN, et al., 2016).

Embora a prevenção da hipertensão seja alcançada com medidas simples como manutenção do peso adequado, mudança dos hábitos alimentares e práticas de atividades físicas, o índice de casos de hipertensão permanece elevado podendo levar a 388 óbitos por dia no Brasil, segundo dados do Sistema de Informações de Mortalidade (SIM), do Ministério da Saúde. A alta prevalência dessa doença mostra que há um déficit na realização da prevenção da mesma (PARANÁ, 2018).

Diante disso, o presente artigo objetiva identificar a atuação da enfermagem, diante dos desafios na prevenção da Hipertensão Arterial na Atenção Primária de Saúde. Percebe-se que há muitos desafios a serem enfrentados pelos profissionais enfermeiros no que diz respeito à prevenção dessa doença, pois, embora seja uma doença comum e bastante conhecida por grande parte das pessoas, sua prevenção e controle ainda é um desafio para a enfermagem. 


\section{MÉTODOS}

Trata-se de uma pesquisa transversal, de caráter descritivo com abordagem qualitativa, desenvolvida na Atenção Primária de Saúde, contemplando quatro Unidades Básicas de Saúde (UBS), sendo elas: UBS 1, UBS 2, UBS 3 e UBS 4, situadas em um munícipio do estado do Maranhão.

A população pesquisada constituiu-se de 12 enfermeiros que atuavam na atenção primária de saúde e que aceitasse participar do estudo assinando o Termo de Consentimento Livre e Esclarecido (TCLE). Os dados foram coletados através de um questionário sobre o respectivo tema com perguntas fechadas. $O$ questionário foi aplicado no consultório e teve duração de 30 minutos. Os participantes foram informados a respeito das objetivações do trabalho e, da autorização para a realização deste estudo através da assinatura do TCLE.

Os resultados foram analisados segundo a técnica de análise de conteúdo de Bardin e foram organizados em categorias (BARDIN L, 1977; SILVA, AH e FOSSÁ, MIT, 2015). A pesquisa foi submetida e aprovada com os princípios éticos e legais do Comitê de Ética em Pesquisa (CEP) com Seres Humanos da Universidade Estadual do Maranhão, campus Caxias, sob parecer 3.783.878.

\section{RESULTADOS E DISCUSSÃO}

Para possibilitar a análise e discussão organizamos os resultados nas seguintes categorias Caracterizando os sujeitos; principais desafios da enfermagem na prevenção da HA; Conhecimento da enfermagem quanto aos fatores de risco da HAS; Atuação da enfermagem quanto aos fatores de risco da Hipertensão e Programas de incentivo a alimentação saudável e atividade física realizados nas UBS.

Quanto a caracterização dos sujeitos das pesquisas buscou-se identificar as variáveis referentes ao sexo e idade. A distribuição de frequência dos enfermeiros segundo o sexo era de onze pessoas do sexo feminino (91,66 \%), um do sexo masculino (8,34\%), totalizando doze enfermeiros.

Com relação à faixa etária, a idade dos enfermeiros deste estudo variou de 25 a 61 anos. A maioria dos enfermeiros encontrava-se com idade entre 30 a 39 anos (66,66 \%), com idade de 24 anos apenas uma enfermeira (8,34), e com idade superior a 50 anos encontrou-se três enfermeiras $(25 \%)$.

\section{Principais desafios da enfermagem na prevenção da HAS}

Esta categoria descreve o conhecimento prévio dos sujeitos sobre os principais desafios dos enfermeiros nesta conduta.

"Conscientizar o paciente quanto à importância da atividade física, uso contínuo da medicação e alimentação saudável" (Enf.7).

"um dos desafios que mais encontramos é fazer com que o paciente leve uma vida de qualidade com alimentação saudável e atividades físicas" (Enf.3).

"orientar toda a população em relação aos riscos da HA" (Enf.4).

"Pacientes com dificuldade de aderir a estilo de vida saudável-alimentação e atividade física" (Enf.8).

"dificuldade em trazer o paciente até a ubs, para realização de consultas principalmente homens, tabagismo, alcoolismo, má alimentação e sedentarismo" (Enf.12).

Diante do exposto é possível identificar que a enfermagem possui conhecimento dos fatores que dificultam a prevenção dessa doença, como por exemplo a dificuldade em adotar hábitos alimentares saudáveis. Esse resultado corrobora com o estudo de Lindemann IL, et al. (2016) onde do total de entrevistados, $(31,1 \%)$ percebiam dificuldades para ter uma alimentação saudável. Dentre os motivos pelos quais referiram dificuldades, $57,6 \%$ mencionaram o custo elevado dos alimentos considerados saudáveis, $49,4 \%$ a força de vontade insuficiente, $41,6 \%$ a necessidade de abrir mão de alimentos considerados não saudáveis, $35,7 \%$ a falta de tempo e $31,3 \%$ o conhecimento insuficiente. 
Outra dificuldade citada pelos enfermeiros é a dificuldade em conscientizar a população a praticar atividade física. Corroborando com o estudo de Carvalho DA, et al. (2017) onde mostra em seu estudo que $90,4 \%$ dos entrevistados acham importante a prática de atividade física, porem $74,8 \%$ destes, não praticava nenhum tipo de exercício físico. Os motivos que levam a falta de atividades físicas são: falta de vontade, problemas visuais, e falta de companheiro $(24,3 \%)$.

Outro resultado encontrado foi a dificuldade da população em procurar os serviços de saúde e que a maioria das pessoas, principalmente os homens só procuram ajuda profissional quando estão apresentando alguns sintomas. O que coincide com o estudo de Cruz PKR, et al. (2020) onde o resultado de sua pesquisa aponta as dificuldades de acesso aos serviços de saúde que são elas: falta de transporte $(30 \%)$, falta de recursos financeiros $(24,6 \%)$, ausência de companhia $(23,1 \%)$, percepção de que o serviço era ruim $(44,6 \%)$, barreiras geográficas $(21,5 \%)$.

A ausência de políticas públicas voltadas ao combate à fome e pobreza, citada por um enfermeiro (8,33 $\%$ ), leva alguma parcela da população a manter-se nos grupos de risco. A pobreza é caracterizada como falta de acesso a bens materiais e oportunidades que geram uma grande vulnerabilidade frente a imprevistos e é um fator de risco para o desenvolvimento de doenças crônicas. Percebe-se que características socioeconômicas dos indivíduos determinam seu estilo de vida e este pode ser um obstáculo para a mudança de comportamento. Ou seja, eles vivem com privações financeira, falta de serviços públicos e infraestrutura de moradia precária, o que leva a dificuldade em aderir um estilo de vida saudável (SOCIEDADE BRASILEIRA DE CARDIOLOGIA, 2016).

\section{Fatores de risco da Hipertensão}

Segundo análise dos questionários pode-se identificar o conhecimento dos enfermeiros quanto aos fatores de risco para a hipertensão.

"muitos pacientes tem uma vida sedentária, a obesidade é um dos principais fatores que encontramos em nossa comunidade, além do uso excessivo de álcool e fumo" (Enf.3).

"Sedentarismo precoce, ausência de atividade física ou esportiva como uma necessidade diária, alimentação fora do horário e ou gordurosa, frituras, doces, consumo de bebidas alcoólicas e fumo" (Enf.7).

"fatores socioeconômico, sedentarismo, falta de informação, má alimentação" (Enf.9).

"perda de peso, tabagismo, sedentarismo, alcoolismo" (Enf.8).

Obesidade e má alimentação e sua relação com a hipertensão, relativo a essa temática, pôde-se detectar que oito $(66,66 \%)$ dos respondentes consideram a má alimentação e obesidade fatores de risco para a hipertensão. Uma alimentação saudável ajuda a proteger contra a má nutrição em todas as suas formas, bem como contra as doenças crônicas não transmissíveis (DCNT), entre elas hipertensão, diabetes, doenças cardiovasculares, AVC e câncer (BRASIL, 2019).

Esse resultado corrobora com a pesquisa de Nilson EAF, et al. (2020), onde afirma que o excesso de peso e a obesidade são fatores de risco importantes para hipertensão e diabetes. A obesidade é um fator predisponente para o desenvolvimento da hipertensão arterial, sendo responsável por $20 \%$ a $30 \%$ dos casos da pressão arterial elevada. As condições socioeconômicas contribuem plenamente na dificuldade de prevenção da hipertensão, uma vez que para ter um estilo de vida saudável é necessário dispor de recursos financeiros que a maioria das pessoas não possui.

Os fatores socioeconômicos e níveis baixos de escolaridade estão diretamente relacionados com o aparecimento de hipertensão. Além de serem fatores de maior risco de aparecimento de lesão em órgãosalvo e eventos cardiovasculares. A baixa renda colabora para a não aderência ao estilo de vida saudável, pois dificulta $O$ acesso a alimentos adequados, a frequentar academias, entre outros (SOCIEDADE BRASILEIRA DE CARDIOLOGIA, 2016). 
Relacionado ao histórico familiar dois enfermeiros (16,66 \%) citaram como um empecilho na prevenção da hipertensão, uma vez que este fator genético está entre os fatores de risco não modificáveis para a HAS. Esse resultado encontra semelhança com o estudo de Jardim TSV, et al. (2015) onde os resultados encontrados indicam que descendentes de indivíduos hipertensos têm maior resistência à insulina, alterações no ritmo circadiano da pressão arterial. Esses achados indicam que jovens saudáveis, com predisposição hereditária para HA, já apresentam alterações metabólicas, de morfologia cardíaca e do comportamento da pressão arterial.

No que tange o sedentarismo seis enfermeiros (50\%) afirmaram que a falta de atividade física é um dos vilões no aparecimento de HAS, e que tem muita dificuldade em fazer com que a população adote o hábito de praticar atividades físicas regulares.

Esse resultado corrobora com o estudo de Carvalho CJ, et al. (2016) em que o sedentarismo esteve presente em $77 \%$ da população estudada, não havendo diferença entre homens e mulheres. A falta de atividade física pode aumentar o risco de desenvolvimento da hipertensão bem como elevar a morbimortalidade desses pacientes.

É reconhecida a importância da atividade física na prevenção de doenças crônicas como a HAS. Porém, é necessário conscientizar a população quanto aos benefícios de praticar atividades físicas e aderir a um estilo de vida mais saudável. Contudo é notório que a população mesmo ciente de tais benefícios, não tem o habito de se exercitar (SOCIEDADE BRASILEIRA DE CARDIOLOGIA, 2016).

Relativo ao efeito do álcool e nicotina sobre a pressão arterial, cinco dos respondentes $(41,66 \%)$ afirmaram serem essas substâncias são um risco para o aumento pressórico, pois eleva temperatura do corpo e aumenta o metabolismo, afetando o sistema cardíaco. O alcoolismo é fator importantíssimo que dificulta a realização da prevenção da hipertensão, uma vez que os profissionais têm dificuldades em fazer com que os pacientes larguem esse vício.

Corroborando com o estudo de Mussi FC, et al. (2018) onde em sua pesquisa feita com hipertensos $37,7 \%$ eram fumantes passivos, 34,6\% ex-tabagistas e 7,7\% fumavam, predominando o uso de cigarro. E quanto a bebida alcoólica $52,3 \%$ dos homens consumiam bebida alcoólica, $(18,5 \%)$ nunca beberam, $(29,2 \%)$ tinham parado.

O tabagismo e alcoolismo são considerados fatores cruciais que dificulta a prevenção da HA. A ingestão demasiada de bebidas alcoólicas eleva consideravelmente a pressão arterial, bem como com o risco de doença cardiovascular. O tabagismo é considerado fator de risco para as doenças cardiovasculares pois induz a vasoconstricção das artérias coronárias tornando-as menos elásticas (MUSSI FC, et al., 2018).

Atualmente existem diversas estratégias assistenciais para conscientizar a população quanto à cessação do uso do tabaco, que incluem abordagem cognitiva-comportamental e uso de medicamentos. $O$ abandono do tabaco pode ser uma experiência difícil e poucos fumantes conseguem interromper com sucesso o hábito de fumar em sua primeira tentativa pois depende de um conjunto de fenômenos comportamentais, cognitivos, sociais e fisiológicos (JESUS MCP, et al., 2016).

A ingestão de álcool é identificada como um fator de risco que contribui para o aparecimento e agravamento da HAS, sua ingestão por períodos prolongados de tempo pode aumentar a pressão arterial e a mortalidade por doenças cardiovasculares (SOCIEDADE BRASILEIRA DE CARDIOLOGIA, 2016).

\section{Orientações de enfermagem quanto aos fatores de risco}

Relativo às orientações necessárias ao controle e prevenção dos fatores de risco observou-se que a equipe de enfermagem presta as devidas orientações tanto nas consultas individuais quanto em ações em grupos específicos.

"orientar quanto à importância de hábitos saudáveis como consumo de água no dia-a-dia 21 por dia, prática de atividade física, dieta hipossódica, eliminar vícioscigarro, álcool” (Enf.7). 
"estimulo a realização de atividade física, perda de peso, controle do colesterol, triglicerídeos- alimentação saudável" (Enf.8).

"explicar os benefícios de uma alimentação balanceada traz pra saúde, incentivo quanto ao uso continuo da medicação, visitas periódicas a ubs para controle da PA." (Enf.9).

Todos os enfermeiros entrevistados afirmaram que uma das principais ferramentas utilizadas para prevenir a HAS é a educação em saúde, fazendo uso de palestras, rodas de conversas, busca ativa de pacientes com fatores de risco, dentre outras ferramentas de educação. É notório que as ações de educação em saúde realizada pelos enfermeiros não atingem totalmente toda a população adscrita. Uma vez que uma equipe é responsável por um número relativamente alto de famílias e atingir diretamente cada uma delas torna-se um pouco difícil. Essa dificuldade também se deve ao fato de que a população não tem conhecimento sobre as ações ou se tem conhecimento não demonstra interesse em participar das mesmas.

Segundo um estudo feito por Kessler M, et al. (2018) Uma das principais estratégias desenvolvidas nas unidades básicas de saúde são os grupos de apoio ao autocuidado realizada por $68,9 \%$ das equipes pesquisadas, realizam essas ações e garantem que a mesma pode estimular processos de educação em saúde, facilitar a construção de vínculos e promover o autocuidado e a adesão ao tratamento. No entanto, a participação nesta atividade é por vezes colocada pelos profissionais como uma exigência ou uma barreira para acesso a determinados serviços, como o acesso à medicação para hipertensão e diabetes.

Quanto à visita domiciliar, ferramenta utilizada pelos enfermeiros no âmbito da atenção primária, caracteriza-se como umas das principais medidas preventivas da hipertensão, pois visa identificar os pacientes que se encontra em risco de desenvolvimento desta doença. Os enfermeiros afirmaram que são realizadas duas vezes por semana visitas domiciliares a população adscrita que não podem, por alguma razão, se deslocar até a unidade. Bezerra MIC, et al. (2015) afirma que através da visita domiciliar é possível conhecer o cotidiano dos indivíduos. Percebemos modificações nos hábitos alimentares, uso correto de medicações, prática de atividades físicas e olhar crítico para prevenção de quedas dentro do domicílio.

A visita domiciliar proporciona contato da equipe de saúde com o paciente fora da UBS. Através dela os profissionais podem conhecer o ambiente familiar, e suas condições de vida. Outras vantagens da atenção domiciliar são maior conforto e tranquilidade para o paciente e família, custos financeiros reduzidos (BEZERRA MIC, et al., 2015).

\section{Programas de incentivo à alimentação saudável e atividade física}

Quantos aos programas de incentivo à alimentação saudável e atividade física foram encontrados o Programa Crescer Saudável, NASF e Feliz Idade. O principal objetivo dos programas é incentivar a população a manter hábitos de vida mais saudáveis.

"Sim. É feito acompanhamento com nutricionista e educador físico, além de consultas periódicas com os demais profissionais" (Enf.4).

"Sim, programa crescer saldável nas escolas e Feliz Idade para hipertensos da comunidade" (Enf.7).

"Sim, palestras na unidade de saúde com a nutricionista e enfermeiros, sobre prevenção e tratamento" (Enf-12).

"Temos nutricionista e orientador físico- NASF" (Enf.10).

Como medida de incentivo a alimentação saudável cinco dos enfermeiros $(41,66)$ afirmaram que é utilizada o Programa Crescer Saudável, um programa destinado as crianças cujo objetivo é incentivar a adoção de hábitos de vida saudáveis como alimentação balanceada e práticas de atividades físicas. É realizado principalmente em escolas com ações especificas para as crianças. Com este programa é possível ensinar as crianças desde cedo à importância de se alimentar bem e com isso conscientizá-los a crescer saudável tornando-se um adulto mais resistente as patologias. 
Este resultado corrobora com o apresentado por Cunha LF (2014) onde afirma que a construção de hábitos saudáveis se configura como um fator de prevenção de futuras doenças, sendo neste caso ainda considerada como o melhor remédio. É de suma importância envolver professores, pais de alunos, profissionais da saúde nas atividades de educação alimentar e nutricional.

O programa Crescer Saudável, criado pelo Ministério da Saúde é constituído de um conjunto de ações articuladas que serão implementadas na Rede de Atenção à Saúde do SUS com objetivo de realizar um acompanhamento adequado do crescimento e desenvolvimento na infância, de modo que possa controlar e tratar a obesidade infantil. O programa é composto por: incentivo a alimentação adequada e saudável, incentivo às práticas corporais e de atividade física (BRASIL, 2019).

Todos os doze entrevistados (100\%), afirmaram fazer uso do Núcleo Ampliado de Saúde da Família e Atenção Básica (NASF-AB), como ferramenta de controle da hipertensão. No programa temos a presença do nutricionista atuando no fornecimento de orientações e incentivando a população a manter uma alimentação saudável como também o educador físico promovendo praticas corporais. O NASF-AB tem como objetivo a integralidade do cuidado aos usuários, ou seja, a saúde depende de fatores além dos biológicos, com isso a atuação dos profissionais de saúde deve levar em conta tais fatores e intervir (SEUS TLC, et al., 2019).

Esse resultando está de acordo com o estudo de Seus TLC, et al. (2019) onde afirma que o programa de atividade física promovido por equipe de NASF fornece benefícios físicos, sociais, psicológicos e econômicos. A atividade física é vista como um tratamento não farmacológico capaz de prevenir a necessidade de utilização dos serviços de saúde, reduzir os gastos com medicamentos e proporcionar bem-estar, sem custos para os participantes.

Dentre os entrevistados seis (50\%) responderam que em sua UBS fazem uso do Programa Feliz Idade que consiste em um projeto direcionado a comunidade idosa, atuando desenvolvendo atividades como: alongamento, caminhada, recreações e demais atividades que auxiliam na saúde e qualidade de vida.

Também são realizados controles de saúde tais como, aferições de pressão, pesagem e testes de glicemia. Esse programa traz diversos benefícios à população idosa tanto melhorias físicas como mentais, proporcionando mais autonomia aos idosos. Os enfermeiros afirmaram ser muito importante esse projeto mediante a prevenção da hipertensão uma vez que a idade é considerada um fator de risco, e a partir das ações realizadas nesse projeto consegue-se prevenir a tal patologia.

Esse resultado também foi encontrado no estudo de Camboim FEF, et al. (2017) onde foi possível observar melhorias na vida e cotidiano das idosas que participaram do programa. As mesmas participantes relataram que houve diminuição das dores musculares e ósseas, que se exercitar diariamente proporciona liberdade para realizar suas tarefas rotineiras. As idosas afirmaram que é de suma importância se manterem ativas devido ao medo de perderem os movimentos e tornarem-se dependentes de uma cadeira de rodas.

\section{CONCLUSÃO}

A enfermagem possui conhecimentos suficientes para orientar os pacientes a realizarem a prevenção da hipertensão. Contudo a população apresenta bastante resistência em seguir as tais orientações, pois mesmo possuindo consciência sobre os hábitos de vida inadequados, sentem-se desmotivados às mudanças. Essa dificuldade advém de algumas barreiras como dificuldade para mudar hábitos, falta de tempo e condições financeiras. De modo geral, as pessoas não conseguem seguir tais condutas de controle, e, sobretudo, adotar um estilo de vida mais saudável, devido à manutenção de hábitos antigos e déficit de conhecimentos. A partir dos resultados e consequentemente dos conhecimentos adquiridos, a equipe poderá intervir diretamente nas fragilidades encontradas, promovendo medidas preventivas mais eficazes e efetivas na comunidade.

\section{REFERÊNCIAS}

1. BARDIN, L. Análise de Conteúdo. Lisboa: Edições 70, 1977.

2. BRASIL. Ministério da Saúde. Política Nacional de Promoção da Saúde: PNPS: Anexo I da Portaria de Consolidação ํo 2, de 28 de setembro de 2017, que consolida as normas sobre as políticas nacionais de saúde do SUS. - Brasília: Ministério da Saúde, 2018. Disponível em:

http://bvsms.saude.gov.br/bvs/publicacoes/politica_nacional_promocao_saude.pdf acesso em: 14 de junho de 2019. 
3. BRASIL. Ministério da Saúde. Secretaria de atenção primária a saúde. Programa Crescer Saudável 2019/2020 Instrutivo. Brasília, 2019. Disponível em: http://189.28.128.100/dab/docs/portaldab/documentos/pse/instrutivo_crescer_saudavel_2019_2020.pdfa acesso em: 05 de julho de 2019.

4. BEZERRA MIC, et al. A visita domiciliar como ferramenta de cuidado da fisioterapia na estratégia saúde da família. Sanare, 2015; 14(01): 76-80.

5. CAMBOIM FEF, et al. Benefícios da atividade física na terceira idade para a qualidade de vida. Rev enferm UFPE on line, 2017; 11(6):2415-22.

6. CARVALHO CJ, et al. Altas taxas de sedentarismo e fatores de risco cardiovascular em pacientes com hipertensão arterial resistente. Medicina, 2016;49(2): 124-133.

7. CARVALHO DA, et al. Prevalência da prática de exercícios físicos em idosos e sua relação com as dificuldades e a falta de aconselhamento profissional específico. R. bras. Ci. e Mov, 2017; 25(1):29-40

8. CUNHA LF. A importância de uma alimentação adequada na educação infantil. Monografia de especialização. Universidade Tecnológica Federal do Paraná, 2014, 32p.

9. CRUZ PKR, et al. Dificuldades do acesso aos serviços de saúde entre idosos não institucionalizados: prevalência e fatores associados. Rev. Bras. Geriatr. Gerontol. 2020; 23(6): 1-13.

10. DANTAS RCO, RONCALLI AG. Protocolo para indivíduos hipertensos assistidos na Atenção Básica em Saúde. Ciênc. saúde coletiva [online], 2019; 24(1):295-306.

11. JARDIM TSV, et al. Influência da hereditariedade em marcadores de risco para hipertensão arterial. Rev Bras Hipertens, 2015; 22(2): 65-71.

12. JESUS MCP, et al. Compreendendo o insucesso da tentativa de parar de fumar: abordagem da fenomenologia social. Rev Esc Enferm USP, 2016; 50(1):73-80.

13. KESSLER M. et al. Ações educativas e de promoção da saúde em equipes do programa nacional de melhoria do acesso e da qualidade da atenção básica. Revista Epidemiol. Serv. Saúde, 2018; 27(2): 1-12.

14. LINDEMANN et al. Dificuldades para alimentação saudável entre usuários da atenção básica em saúde e fatores associados. Revista Ciênc. saúde coletiva, 2016; 21(2): 599-610.

15. MALTA DC, et al. Prevalência da hipertensão arterial segundo diferentes critérios diagnósticos, Pesquisa Nacional de Saúde. Ver. Bras. Epidemiol. 2018;21(1): 1-15.

16. MENEZES TN, et al. Prevalência e controle da hipertensão arterial em idosos: um estudo populacional. Revista Portuguesa de Saúde Pública. 2016; 34 (2):117-124.

17. MUSSI FC, et al. Consumo de bebida alcoólica e tabagismo em homens hipertensos. Rev baiana enferm 2018; $32: 1$ 12.

18. NILSON EAF, et al. Custos atribuíveis a obesidade, hipertensão e diabetes no Sistema Único de Saúde, Brasil, 2018. Rev Panam Salud Publica, 2020; 44-32.

19. REIS LLM, et al. Métodos não farmacológicos utilizados pelo enfermeiro na prevenção e controle da hipertensão arterial sistêmica. Revista Nursing, 2018; 21(1): 2338-2341.

20. SEUS TLC, et al. NASF: promoção da saúde e atividade física - PMAQ 2013. Epidemiol. Serv. Saude, 2019; 28(2): 114.

21. SILVA, AH; FOSSÁ, MIT. Análise de conteúdo: exemplo de aplicação da técnica para análise de dados qualitativos. Qualitas Revista Eletrônica, 2015; 17(1): 1-14.

22. SOCIEDADE BRASILEIRA DE CARDIOLOGIA. VII Diretrizes Brasileiras de Hipertensão, 2016. Disponível em: http://publicacoes.cardiol.br/2014/diretrizes/2016/05_HIPERTENSAO_ARTERIAL.pdf. Acessado em: 20 de abril de 2019.

23. PARANÁ. Linha guia de hipertensão Arterial, $2018 . \quad$ Disponível em: http://www2.maringa.pr.gov.br/sistema/arquivos/db5be589f90e.pdf acesso em 10 de maio de 2019.

24. PEREIRA DS, et al. A atividade física na prevenção das doenças crônicas não transmissíveis. Revista Gestão e Saúde. $2017 ; 1: 1-9$. 